secretion in response to a variety of stimuli (1). The reduced insulin secretion in turn explains the increased hepatic glucose production observed. There are still many unanswered questions: Is the concentration of TCF7L2 protein increased in the $\beta$ cell in addition to TCF7L2 RNA? How is expression increased by intronic variants? How does increased TCF7L2 expression reduce insulin secretion? This is the first in a series of steps toward understanding the associated pathophysiology. In the end, what is desired from scientific breakthroughs is improved prevention of type 2 diabetes and improved treatment of those who develop the disease. We are still a long way from this, but there is now a new $\beta$ cell pathway to be further investigated to see if it can be manipulated by drugs or lifestyle changes.

\section{Acknowledgments}

Andrew T. Hattersley is a Wellcome Trust Research Leave Fellow.

Address correspondence to: Andrew T. Hattersley, Peninsula Medical School, Barrack Road, Exeter, EX2 5DW, United Kingdom. Phone: 44-1392-406806; Fax: 44-1392-406767; E-mail: Andrew.Hattersley@pms.ac.uk.

1. Lyssenko, V., et al. 2007. Mechanisms by which common variants in the $T C F 7 L 2$ gene increase risk of type 2 diabetes. J. Clin. Invest. 117:2156-2163. doi: $10.1172 / \mathrm{JCI} 30706$.
2. Altshuler, D., et al. 2000. The common PPARgamma Pro12Ala polymorphism is associated with decreased risk of type 2 diabetes. Nat. Genet. 26:76-80.

3. Gloyn, A.L., et al. 2003. Large-scale association studies of variants in genes encoding the pancreatic beta-cell K-ATP channel subunits Kir6.2 (KCNJ11) and SUR1 $\mathrm{ABCC} 8$ ) confirm that the KCNJ11 E23K variant is associated with Type 2 diabetes. Diabetes. 52:568-572.

4. Barroso, I., et al. 1999. Dominant negative mutations in human PPARgamma associated with severe insulin resistance, diabetes mellitus and hypertension. Nature. 402:880-883.

5. Gloyn, A.L., et al. 2004. Activating mutations in the gene encoding the ATP-sensitive potassium-channel subunit Kir6.2 and permanent neonatal diabetes. N. Engl. J. Med. 350:1838-1849.

6. Grant, S.F., et al. 2006. Variant of transcription factor 7-like 2 (TCF7L2) gene confers risk of type 2 diabetes. Nat. Genet. 38:320-323.

7. Zeggini, E., and McCarthy, M.I. 2007. TCF7L2: the biggest story in diabetes genetics since HLA? Diabetologia. 50:1-4.

8. Chandak, G.R., et al. 2007. Common variants in the TCF7L2 gene are strongly associated with type 2 diabetes mellitus in the Indian population. Diabetologia. 50:63-67.

9. Hayashi, T., Iwamoto, Y., Kaku, K., Hirose, H., and Maeda, S. 2007. Replication study for the association of TCF7L2 with susceptibility to type 2 diabetes in a Japanese population. Diabetologia. 50:980-984.

10. Horikoshi, M., et al. 2007. A genetic variation of the transcription factor 7 -like 2 gene is associated with risk of type 2 diabetes in the Japanese population. Diabetologia. 50:747-751.

11. Lehman, D.M., et al. 2007. Haplotypes of transcription factor 7-like 2 (TCF7L2) gene and its upstream region are associated with type 2 diabetes and age of onset in Mexican Americans. Diabetes. 56:389-393.

12. Helgason, A., et al. 2007. Refining the impact of TCF7L2 gene variants on type 2 diabetes and adaptive evolution. Nat. Genet. 39:218-225.

13. Zeggini, E., et al. 2007. Replication of genome-wide association signals in UK samples reveals risk loci for type 2 diabetes. Science. 316:1336-1341.

14. Sladek, R., et al. 2007. A genome-wide association study identifies novel risk loci for type 2 diabetes. Nature. 445:881-885.

15. Scott, L.J., et al. 2007. A genome-wide association study of type 2 diabetes in Finns detects multiple susceptibility variants. Science. 316:1341-1345.

16. Saxena, R., et al. 2007. Genome-wide association analysis identifies loci for type 2 diabetes and triglyceride levels. Science. 316:1331-1336.

17. Steinthorsdottir, V., et al. 2007. A variant in CDKAL1 influences insulin response and risk of type 2 diabetes. Nat. Genet. 39:770-775.

18. Horikawa, Y., et al. 2000. Genetic variation in the gene encoding calpain-10 is associated with type 2 diabetes mellitus. Nat. Genet. 26:163-175.

19. Weedon, M.N., et al. 2003. Meta-analysis and a large association study confirm a role for calpain10 variation in type 2 diabetes susceptibility. Am.J. Hum. Genet. 73:1208-1212.

20. Tsuchiya, T., et al. 2006. Association of the calpain10 gene with type 2 diabetes in Europeans: results of pooled and meta-analyses. Mol. Genet. Metab. 89:174-184.

21. Johnson, J.D., et al. 2004. RyR2 and calpain-10 delineate a novel apoptosis pathway in pancreatic islets. J. Biol. Chem. 279:24794-24802.

22. Saxena, R., et al. 2006. Common single nucleotide polymorphisms in TCF7L2 are reproducibly associated with type 2 diabetes and reduce the insulin response to glucose in nondiabetic individuals. Diabetes. 55:2890-2895.

23. Freathy, R.M., et al. 2007. Type 2 diabetes TCF7L2 risk genotypes alter birth weight: a study of 24,053 individuals. Am. J. Hum. Genet. 80:1150-1161.

24. Florez, J.C., et al. 2006. TCF7L2 polymorphisms and progression to diabetes in the Diabetes Prevention Program. N. Engl. J. Med. 355:241-250.

25. Loos, R.J., et al. 2007. TCF7L2 polymorphisms modulate proinsulin levels and beta-cell function in a British Europid population. Diabetes. 56:1943-1947.

\title{
Proteinuria: is it all in the foot?
}

\section{Pierre Ronco}

INSERM UMR S 702, University Pierre et Marie Curie, and Department of Nephrology and Dialysis, Tenon Hospital, Paris, France.

\begin{abstract}
Despite significant advances in our understanding of the molecular structure and composition of the glomerular filtration barrier, the mechanisms underlying the presence of excess protein in the urine (proteinuria) in acquired human kidney diseases remain elusive. In a study appearing in this issue of the JCI, Sever and associates use a combination of biochemical, genetic, and in vivo approaches in mice to demonstrate a pivotal role of cathepsin L and its substrate the GTPase dynamin, in the induction of proteinuria and associated foot process effacement in glomerular podocytes (see the related article beginning on page 2095).
\end{abstract}

Nonstandard abbreviations used: AngII, angiotensin II; CatL, cathepsin L; CD2AP, CD2-associated protein; FSGS, focal segmental glomerular sclerosis; GBM, glomerular basement membrane; MCD, minimal change disease; Neph1, nephrin 1; SD, slit diaphragm.

Conflict of interest: The author has declared that no conflict of interest exists.

Citation for this article: J. Clin. Invest. 117:2079-2082 (2007). doi:10.1172/JCI32966.
One main function of the kidney is to filter low-molecular-weight plasma waste products into the urine while restricting the passage of albumin and larger molecules that are necessary for maintaining normal homeostasis. This occurs in special filtration units called glomeruli, which consist of a capillary tuft inside the Bowman cap- sule and every day form about 180 liters of primary urine virtually devoid of macromolecules. The glomerular capillary wall is characterized by an extremely high permeability to water, small solutes, and ions, contrasting with a very low permeability to plasma proteins of the size of albumin and larger. The essential components of the glomerular capillary wall are the fenestrated endothelium, the glomerular basement membrane (GBM), and the podocytes (Figure 1). The latter cells have a voluminous body and long primary processes that extend toward the capillaries to which they affix by numerous foot processes. The foot processes of neighboring podocytes regularly interdigitate, forming filtration slits between them that are bridged by slit 

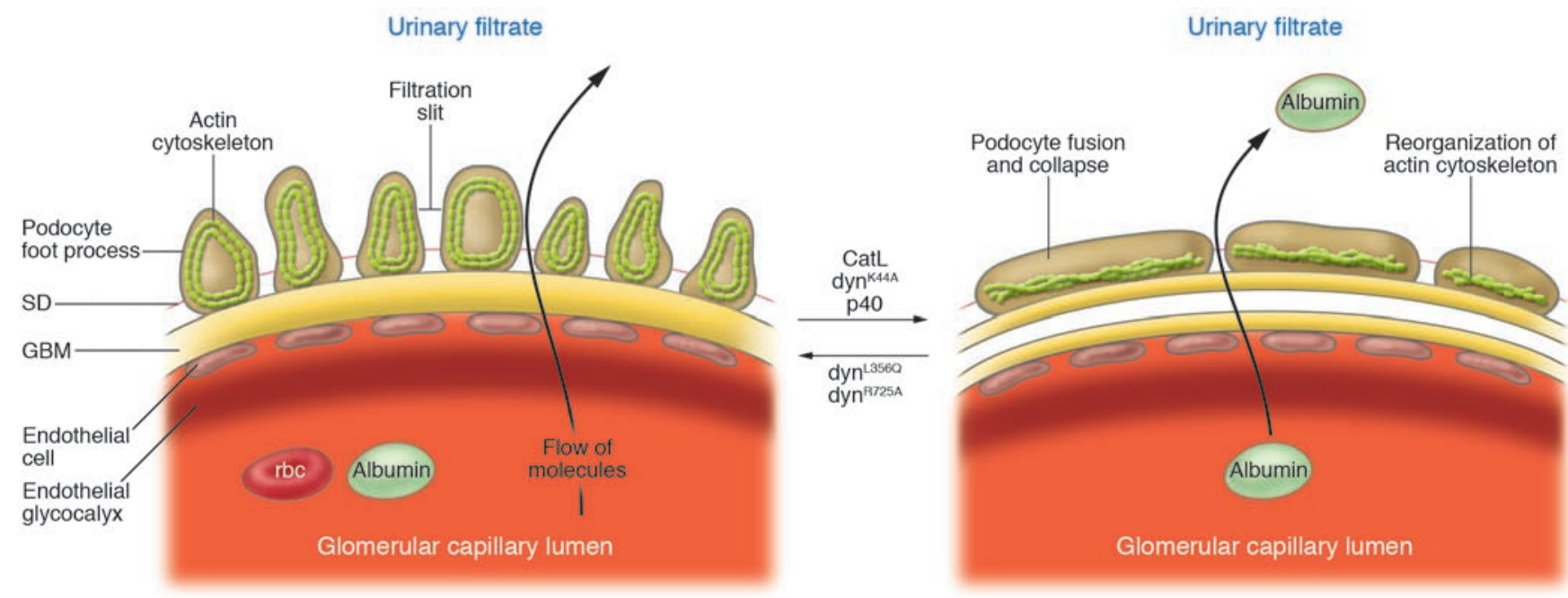

\section{Figure 1}

The glomerular filtration barrier. Blood enters the glomerular capillaries and is filtered across the endothelium and the glomerular basement membrane and through the filtration slits between podocyte foot processes to produce the primary urine filtrate. In healthy glomeruli, this barrier restricts the passage of macromolecules. In this issue of the $J C l$, Sever et al. (10) show that CatL, the expression of which is increased in human proteinuric diseases and in an LPS-induced mouse model of proteinuria, causes proteinuria and foot process effacement through cleavage of the GTPase dynamin, an actin-binding protein. The same effects are induced by gene delivery into mice of dyn ${ }^{\mathrm{K} 44 \mathrm{~A}}-\mathrm{a}$ mutant form of dynamin that does not bind GTP — or of the CatL-cleaved product of dynamin (p40). Conversely, gene delivery into proteinuric mice of dyn ${ }^{\mathrm{L} 356 \mathrm{Q}}$ and dyn ${ }^{\mathrm{R} 725 \mathrm{~A}}$, two CatL-resistant dynamin mutants, reverses proteinuria and foot process effacement. Figure modified from ref. 28.

diaphragms (SDs) with shallow adherens junctions. The structure of the glomerular capillary wall is altered in most kidney diseases, leading to proteinuria. To date, treatment remains mostly empirical, and pathophysiology-driven therapies are lacking.

\section{Role of the SD in hereditary proteinuric disease}

Studies of hereditary proteinuric syndromes have uncovered the presence of transmembrane proteins that participate in the formation of the SD, thus pointing to a role for this structure in preventing protein leakage (Figure 2). Nephrin was the first specialized SD protein identified (1). Molecular modelling and high-resolution electron tomography indicate that nephrin molecules undergo homophilic interactions to form zipper-like sheets with a constant width of approximately $40 \mathrm{~nm}$ and pores the diameter of the albumin molecule or smaller. Other components of the sheets include nephrin 1 (Neph1), Neph2, fatty acid transporter tumor suppressor homolog-1 (FAT1), and FAT2 (reviewed in ref. 2). Nephrin and Neph molecules interact with podocin (3) and CD2-associated protein (CD2AP). Nephrin, podocin, and CD2AP are embedded into lipid rafts (4), where podocin seems to play a key role in facilitating nephrin signaling (5) and activating transient receptor potential cation channel, subfamily $\mathrm{C}$, member 6 (TRPC6) (6). SDs are firmly attached to the actin cytoskeleton through linker proteins, including the catenins, zona occludens 1 (ZO-1), CD2AP, and Nck $(7,8)$ (Figure 2), which likely explains why genetic defects altering SD structure and function are usually associated with actin cytoskeleton reorganization and foot process effacement.

These findings have increased the fascination with the $\mathrm{SD}$, although calculations suggest that the size-selectivity mechanism of the glomerular capillary wall resides solely in the GBM (9). Furthermore, hereditary proteinuric syndromes account for a small percentage of kidney diseases. In the far more common acquired causes of proteinuria, molecular mechanisms of proteinuria and associated foot process effacement are poorly understood. The study by Sever et al. in this issue of the JCI (10) defines a new mechanism underlying proteinuria that primarily affects foot processes.

\section{The actin cytoskeleton as a central organizer}

The major processes are composed of microtubules and vimentin intermediate filaments, while the foot processes are made of actin filaments only. The actin microfilaments form a complex contractile apparatus that plays an important role in maintaining podocyte architecture and counteracting the expansive forces of the capillary. The actin cytoskeleton comprises actin bundles associated with myosin II that run along the longitudinal axis of the foot process above the level of the SD and a cortical actin network that is distributed beneath the foot process plasma membrane (11) (Figure 1). The actin-binding proteins $\alpha$-actinin and synaptopodin are observed in the actin bundles, while cortactin is associated with the cortical actin network (11). Foot processes are attached to the GBM through $\alpha_{3} \beta_{1}$ integrin and dystroglycans, which serve as receptors for GBM components (reviewed in ref. 12). The actin bundles are presumably connected to the integrins through submembranous cytoskeletal proteins (e.g., talin, vinculin, and paxillin) and the cortical actin network. In addition, the actin cytoskeleton associates with anionic podocalyxin through interactions with ezrin and NHERV-2 (Figure 2).

The foot processes are highly dynamic structures that can be reorganized within minutes due to changes in the actin cytoskeleton. Actin filaments, which are organized into coordinated stress fibers in normal foot processes, can reversibly form a dense network with effacement of foot processes and obliteration of filtration slits (Figure 1). Most proteinuric diseases are associated with early foot process effacement. The current belief is that loss 


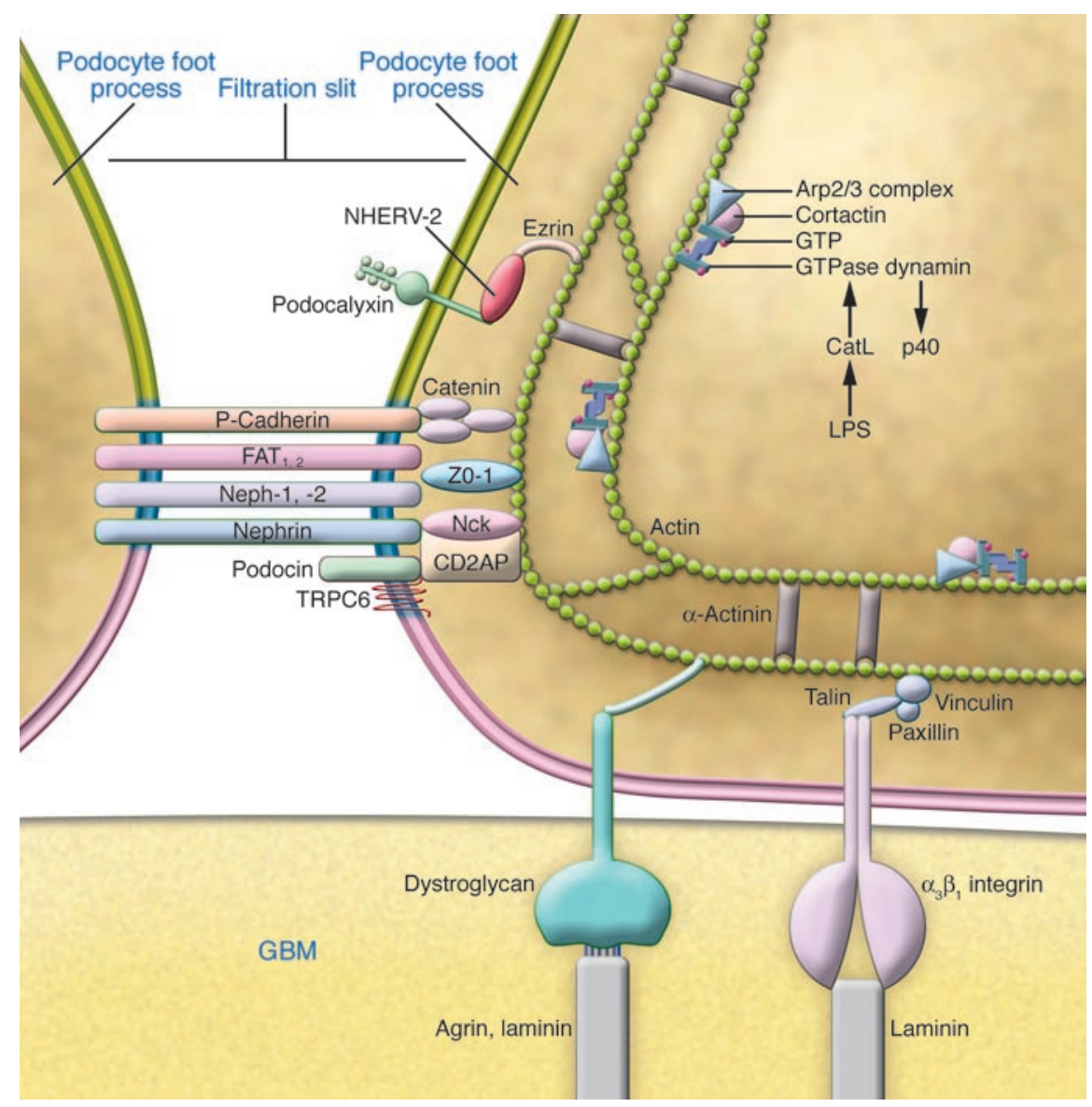

of the organized structure of the actin cytoskeleton is a prerequisite for foot process effacement, ensuing alterations of the SD, and proteinuria (12). Several pathomechanisms can affect the actin cytoskeleton. First, mutation of $\alpha$-actinin 4 , which causes hereditary focal segmental glomerulosclerosis (FSGS), impairs cytoskeletal dynamics (13). A second mechanism involves loss of integrity of the SD complex. The recent findings that Nck adaptor proteins link nephrin to the actin cytoskeleton provide new molecular insights $(7,8)$. According to this model, nephrin becomes phosphorylated during formation of the foot processes, which results in recruitment of Nck and induction of actin polymerization. Once the SD is assembled, nephrin molecules are dephosphorylated and nephrin is connected to actin through CD2AP and podocin. Following SD injury, nephrin molecules become clustered, which induces their phosphorylation, Nck recruitment, actin polymerization, and foot process effacement. Third, foot process effacement can result from alteration in the composition and organization of the GBM, as is the case in laminin $\beta 2$-deficient mice, in which proteinuria precedes foot process effacement (14). More generally, foot processes can be the target of aggressors borne by the circulation or produced locally (e.g., ROS).

\section{Regulation of the actin cytoskeleton by the GTPase dynamin}

In this issue of the JCI, Sever et al. (10) describe a novel mechanism of proteinuria induced by cathepsin L-mediated (CatLmediated) cleavage of the GTPase dynamin, an enzymatic protein that is associated with actin filaments and appears to be closely involved in the remodeling of the actin cytoskeleton (reviewed in ref. 15) (Figure 2). The authors show that CatL levels are increased in human proteinuric kidney diseases and in an LPS mouse model of minimal change disease (MCD). In this model, proteinuria and foot process effacement are abolished in CatL-deficient mice. The authors then identified the GTPase dynamin as a CatL substrate. Dynamin is detected in foot process actin bundles and in the cortical actin cytoskeleton in normal mice, while its expression appears weaker and clustered in

\section{Figure 2}

Pathomechanisms of proteinuria induced by GTPase dynamin cleavage by CatL. This schematic shows the connections between the cortical actin cytoskeleton and components of the basolateral portion of a podocyte. The membrane domains are marked: apical cell membrane (green); the SD domain (blue); and the "sole" of the foot process (pink). In this issue of the $\mathrm{JCl}$, Sever et al. (10) show that LPS injection into mice induces CatL expression in the cytoplasm, which cleaves the active form of GTPase dynamin (represented here in its GTP-bound homotetrameric form). Dynamin binds actin-regulatory proteins including cortactin and the Arp2/3 complex, which catalyze actin filament assembly. Cleavage of dynamin generates a 40-kDa dynamin fragment ( $p 40)$, which interferes with the normal function of dynamin and induces cytoskeleton reorganization, foot process effacement, and proteinuria as shown in Figure 1. Figure adapted from ref. 12. proteinuric animals. Gene delivery of dynamin mutants into cultured podocytes or into mice treated with LPS established that CatL recognizes an evolutionally conserved motif in dynamin and generates a $40-\mathrm{kDa}$ dynamin fragment ( $\mathrm{p} 40$ ) only when dynamin is in the GTP-bound conformation. Sever and associates further showed that gene delivery into mice of the dominantnegative dynamin mutant dyn ${ }^{\mathrm{K} 44 \mathrm{~A}}$ (which cannot bind GTP) or of p40 caused transient proteinuria and foot process effacement. The expression of $\mathrm{dyn}^{\mathrm{K} 44 \mathrm{~A}}$ or $\mathrm{p} 40$ abolished actin stress fibers while enhancing the cortical actin web. Conversely, gene delivery of dynamin mutants that are resistant to CatL cleavage abolished or markedly reduced LPS-induced proteinuria.

Taken together, these studies (10) suggest that dynamin is normally required to maintain the ultrafiltration barrier in glomeruli, possibly by regulating the actin cytoskeleton, and that CatL "switches off" the active, GTP-bound form of dynamin. A well-characterized biochemical link between dynamin and actin filament assembly is cortactin (reviewed in ref. 15) (Figure 2). 
On the other hand, both dynamin and actin are implicated in clathrin-mediated endocytosis (16), which is involved in endocytosis of albumin in nephrotic states and exocytosis of the membrane attack complex of complement in membranous nephropathy (17). Although Sever et al.'s experiments (10) do not favor inhibition of endocytosis by LPS or dynamin mutants, the interplay between actin dynamics and endocytosis needs further clarification.

\section{Pathomechanisms of proteinuria in minimal change disease and FSGS}

MCD is the most frequent cause of acquired nephrotic syndrome in children. It is characterized by massive proteinuria, mostly normal glomeruli as observed by light microscopy, generalized foot process effacement, and corticosteroid responsiveness. MCD is considered a disorder of $\mathrm{T}$ lymphocyte function. A glomerular permeability factor was found in the supernatant of T lymphocytes from MCD patients that induced proteinuria and partial foot process effacement when injected into mice or rats $(18,19)$. In FSGS, circulating factors may account for the recurrence of nephrotic syndrome after renal transplantation. The mechanisms whereby those permeability factors could induce CatL in the glomeruli of patients with FSGS, and to a lesser extent in those with MCD (10), remain elusive. There are several possible targets on podocytes for those circulating factors, including integrins, cell-surface receptors, and SD proteins. Because CatL was induced in podocytes lacking $\alpha 3$ integrin (20), the permeability factor(s) might bind $\alpha_{3} \beta_{1}$ integrin and upregulate CatL.

\section{Pathomechanisms of proteinuria without (initial) foot process effacement}

Massive proteinuria can occur without foot process effacement (reviewed in ref. 21). In mice deficient in GBM laminin $\beta 2$, albuminuria occurred more than seven days before foot process effacement and loss of SD (14). Injection of antibodies to nephrin induced massive proteinuria in rats with morphologically intact SD and well-preserved foot processes (22). Proteinuria without foot process effacement is also observed in women with preeclampsia and severe endothelial lesions (23) and cases of nephrotic syndrome (24, 25). These findings demonstrate that defects in any of the three components of the glo- merular capillary wall can lead to proteinuria without initial foot process effacement. Why is sustained proteinuria usually associated with foot process effacement? In vitro studies suggest that albumin and IgG present at concentrations normally found in the plasma stimulate changes in the podocyte cytoskeleton (26). In vivo, podocytes exhibit high endocytic activity in nephrotic conditions. Whether this endocytic activity induces CatL and dynamin p40 remains to be established.

\section{Therapeutic perspectives}

Sustained proteinuria is a well-established factor of renal disease progression. To date, angiotensin-converting enzyme inhibitors and angiotensin II (AngII) receptor antagonists are the most effective antiproteinuric agents. In addition to their beneficial hemodynamic effects, they may block AngII-induced actin cytoskeleton reorganization (27). Whether this effect involves CatL inhibition and/or active GTPase dynamin stabilization will require further investigation. The use of dynamin mutants and CatL inhibitors as a treatment for proteinuric diseases is a new option that must be further explored.

Address correspondence to: Pierre Ronco, Department of Nephrology and Dialysis, Tenon Hospital, 4 rue de la Chine, 75020 Paris, France. Phone: 33-1-56-01-66-39; Fax: 33-1-56-01-69-99; E-mail: pierre. ronco@tnn.aphp.fr.

1. Kestila, M., et al. 1998. Positionally cloned gene for a novel glomerular protein - nephrin - is mutated in congenital nephrotic syndrome. Mol. Cell. 1:575-582.

2. Tryggvason, K., Patraaka, J., and Wartiovaara, J. 2006. Hereditary proteinuria syndromes and mechanisms of proteinuria. N. Engl. J. Med. 354:1387-1401.

3. Boute, N., et al. 2000. NPHS2, encoding the glomerular protein podocin, is mutated in autosomal recessive steroid-resistant nephrotic syndrome. Nat. Genet. 24:349-354.

4. Schwarz, K., et al. 2001. Podocin, a raft-associated component of the glomerular slit diaphragm, interacts with CD2AP and nephrin. J. Clin. Invest. 108:1621-1629. doi:10.1172/JCI200112849.

5. Huber, T.B., et al. 2001. Interaction with podocin facilitates nephrin signaling. J. Biol. Chem. 276:41543-41546.

6. Huber, T.B., et al. 2006. Podocin and MEC-2 bind cholesterol to regulate the activity of associated ion channels. Proc. Natl. Acad. Sci. U. S. A. 103:17079-17086

7. Verma, R., et al. 2006. Nephrin ectodomain engagement results in Src kinase activation, nephrin phosphorylation, Nck recruitment, and actin polymerization. J. Clin. Invest. 116:1346-1359. doi:10.1172/JCI27414.

8. Jones, N., et al. 2006. Nck adaptor proteins link nephrin to the actin cytoskeleton of kidney podocytes. Nature. 440:818-823.

9. Smithies, O. 2003. Why the kidney glomerulus does not clog: a gel permeation/diffusion hypothesis of renal function. Proc. Natl. Acad. Sci. U. S. A. 100:4108-4113.

10. Sever, S., et al. 2007. Proteolytic processing of dynamin by cytoplasmic cathepsin L is a mechanism for proteinuric kidney disease. J. Clin. Invest. 117:2095-2104. doi:10.1172/JCI32022.

11. Ichimura, K., Kurihara, H., and Sakai, T. 2003. Actin filament organization of foot processes in rat podocytes. J. Histochem. Cytochem. 51:1589-1600.

12. Kerjaschki, D. 2001. Caught flat-footed: podocyte damage and the molecular bases of focal glomerulosclerosis. J. Clin. Invest. 108:1583-1587. doi:10.1172/JCI200114629.

13. Michaud, J.L., et al. 2006. FSGS-associated alphaactinin-4 (K256E) impairs cytoskeletal dynamics in podocytes. Kidney Int. 70:1054-1061.

14. Jarad, G., Cunningham, J., Shaw, A.S., and Miner, J.H. 2006. Proteinuria precedes podocyte abnormalities in Lamb2 ${ }^{-/-}$mice, implicating the glomerular basement membrane as an albumin barrier. J. Clin. Invest. 116:2272-2279. doi:10.1172/JCI28414.

15. Schafer, D.A. 2004. Regulating actin dynamics at membranes: a focus on dynamin. Traffic. 5:463-469.

16. Merrifield, C.J., et al. 2002. Imaging actin and dynamin recruitment during invagination of single clathrin-coated pits. Nat. Cell Biol. 4:691-698.

17. Kerjaschki, D., et al. 1989. Transcellular transport and membrane insertion of the C5b-9 membrane attack complex of complement by glomerular epithelial cells in experimental membranous nephropathy. J. Immunol. 143:546-552.

18. Lagrue, G., et al. 1975. A vascular permeability factor in lymphocyte culture supernatants from patients with nephrotic syndrome. II. Pharmacological and physicochemical properties. Biomedicine. 23:73-75.

19. Koyama, A., et al. 1991. A glomerular permeability factor produced by human $\mathrm{T}$ cell hybridomas. Kidney Int. 40:453-460.

20. Reiser, J., et al. 2004. Podocyte migration during nephrotic syndrome requires a coordinated interplay between cathepsin $\mathrm{L}$ and alpha3 integrin. J. Biol. Chem. 279:34827-34832.

21. Kalluri, R. 2006. Proteinuria with and without renal glomerular podocyte effacement. J. Am. Soc. Nephrol. 17:2383-2389.

22. Topham, P.S., et al. 1999. Nephritogenic mAb 5-1-6 is directed at the extracellular domain of rat nephrin. J. Clin. Invest. 104:1559-1566.

23. Pirani, C.L., Pollak, V.E., Lannigan, R., and Folli, G. 1963. The renal glomerular lesions of pre-eclampsia: electron microscopic studies. Am. J. Obstet. Gynecol. 87:1047-1070.

24. Good, K.S., O’Brien, K., Schulman, G., Kerjaschki, D., and Fogo, A.B. 2004. Unexplained nephroticrange proteinuria in a 38 -year-old man: a case of "no change disease." Am. J. Kidney Dis. 43:933-938.

25. Branten, A.J., et al. 2001. Familial nephropathy differing from minimal change nephropathy and focal glomerulosclerosis. Kidney Int. 59:693-701.

26. Morigi, M., et al. 2005. In response to protein load podocytes reorganize cytoskeleton and modulate endothelin-1 gene: implication for permselective dysfunction of chronic nephropathies. Am. J. Pathol. 166:1309-1320.

27. Macconi, D., et al. 2006. Permselective dysfunction of podocyte-podocyte contact upon angiotensin II unravels the molecular target for renoprotective intervention. Am. J. Pathol. 168:1073-1085.

28. Quaggin, S.E. 2007. Sizing up sialic acid in glomerular disease. J. Clin. Invest. 117:1480-1483. doi:10.1172/JCI32482. 